\title{
Novel and successful free comments method for sensory characterization of chocolate ice cream: A comparative study between pivot profile and comment analysis
}

\author{
Fernando G. A. Fonseca, ${ }^{*}$ Erick A. Esmerino, ${ }^{* 1}$ Elson R. Tavares Filho, ${ }^{*}$ Juliana P. Ferraz, ${ }^{*}$ \\ Adriano G. da Cruz, $†$ and Helena M. A. Bolini* \\ *University of Campinas (UNICAMP), Faculty of Food Engineering (FEA), Department of Food and Nutrition (DEPAN), Campinas, São Paulo, \\ Brazil 13083-862 \\ †Federal Institute of Rio de Janeiro (IFRJ), Department of Food Science and Technology, Rio de Janeiro, Brazil 20260-100
}

\begin{abstract}
Rapid sensory profiling methods have gained space in the sensory evaluation field. Techniques using direct analysis of the terms generated by consumers are considered easy to perform, without specific training requirements, thus improving knowledge about consumer perceptions on various products. This study aimed to determine the sensory profile of different commercial samples of chocolate ice cream, labeled as conventional and light or diet, using the "comment analysis" and "pivot profile" methods, based on consumers' perceptions. In the comment analysis task, consumers responded to 2 separate open questions describing the sensory attributes they liked or disliked in each sample. In the pivot profile method, samples were served in pairs (consisting of a coded sample and pivot), and consumers indicated the higher and lower intensity attributes in the target sample compared with the pivot. We observed that both methods were able to characterize the different chocolate ice cream samples using consumer perception, with high correlation results and configurational similarity (regression vector coefficient $=0.917$ ) between them. However, it is worth emphasizing that comment analysis is performed intuitively by consumers, whereas the pivot profile method showed high analytical and discriminative power even using consumers, proving to be a promising technique for routine application when classical descriptive methods cannot be used.
\end{abstract}

Key words: ice cream, pivot profile, comment analysis, sensory characterization, consumer perception

Received June 18, 2015.

Accepted November 2, 2015.

${ }^{1}$ Corresponding author: erick.almeida@hotmail.com

\section{INTRODUCTION}

Ice cream is a milk-based food consumed worldwide, being a popular semi-solid dairy dessert well accepted among consumers of all ages (Cadena et al., 2012; SunWaterhouse et al., 2013). It is mainly formed by air bubbles, fat globules, and ice crystals dispersed in an aqueous phase of sugars, proteins, minerals, stabilizers, emulsifiers, colorings, and flavorings, besides containing artificial flavorings agents or extracts (Soukoulis and Tzia, 2010; da Silva et al., 2014).

Consumers are becoming increasingly aware of and looking for foods with health benefits. Joint efforts between the food industry and the academic sector have led to studies on changes in ice cream formulations (Cadena and Bolini, 2011). These strategies include fat replacement (Aykan et al., 2008), sucrose substitution (Ozdemir et al., 2015), addition of different types of fiber (Dervişoglu and Yazici, 2006), and use of probiotic cultures (Cruz et al., 2009; Ferraz et al., 2012).

Although technological changes in ice cream have multiple purposes, they must be carefully managed and investigated. Because of interactions among ingredients in a formulation, changes in the nature or amount of ingredients, such as fat or sugar, may result in undesirable changes in the ice cream, with negative effects on the sensory profile of the final product, especially with respect to aroma, flavor, and texture (Frøst et al., 2005).

Understanding how consumers perceive food products is an arduous and challenging task for both food industry and sensory researchers and should not be underestimated. This information is necessary for the development, marketing, and reformulation of existing products, and for establishing specifications for quality control programs (Meilgaard et al., 1999; Ares, 2015).

Quantitative descriptive analysis (QDA) stands out among classic methods of sensory characterization, and 
it yields detailed, robust, consistent, and reproducible results through trained and selected assessors (Cruz et al., 2013). The disadvantage of using trained assessors in relation to consumers is evidenced by the different perceptions and descriptions of sensory attributes. Trained assessors often report attributes irrelevant to consumer perceptions (ten Kleij and Musters, 2003; Symoneaux et al., 2012). Furthermore, the use of a trained panel is costly and time consuming. For a small food company, the routine use of trained assessors can be cost prohibitive, and for large industries, the situation may be even more problematic due to the wide variety of products (Cruz et al., 2013).

Some studies have attempted to investigate and understand consumer perceptions through direct analysis of the mentioned words. These methods use the perception and spontaneous comments of consumers to differentiate and characterize products (Parente et al., 2011; Symoneaux et al., 2012). They exploit the generation of vocabulary and sensory descriptors, using free-listing tasks, open-ended questions, and word association with consequent textual analysis of the data (ten Kleij and Musters, 2003; Ares and Deliza, 2010; Lawrence et al., 2013).

Methods based on free comments are easy to perform, as they do not require specific training and can be applied to consumers (ten Kleij and Musters, 2003). The data collected are a rich source of information and should not be undervalued (ten Kleij and Musters, 2003). When analyzed properly, such data can provide results similar to those obtained through classic sensory characterization and can complement quantitative results (Symoneaux et al., 2012; Lawrence et al., 2013).

Open-ended questions with subsequent comment analysis of likes and dislikes have been successfully used in the sensory characterization of some foods (Galmarini et al., 2013). Consumers are asked to answer 2 open-ended questions indicating what they liked and disliked about each sample after giving their overall liking score. Comment analysis involves a frequency count of the sensory attributes used to characterize a product, generating a contingency table (Lawrence et al., 2013). The recent use of the chisquare test per cell has made it possible to increase the accuracy of data interpretation, and it has proven to be an interesting tool for sensory differentiation and, perhaps, an alternative to preference mapping (Symoneaux et al., 2012).

When free descriptions are used, as in open-ended questions, an association between an attribute and its intensity can occur, even when samples are presented in a monadic manner. In that case, data interpretation is a critical procedure and should be conducted carefully (ten Kleij and Musters 2003; Giboreau et al., 2009). Erroneous and unrepresentative results can be generated by considering all degrees of difference, besides decreasing refinement and statistical power. Pivot profile $(\mathbf{P P})$ aimed to reduce this limitation was recently proposed by Thuillier et al. (2015), although its use is still limited.

Pivot profile is a descriptive method recently introduced that has been used in the characterization of Champagne varieties by experts. The PP task uses a comparative format between the target product and another product called the "pivot." The related responses assume the form of "less" or "more" intense than the pivot, minimizing variability within assessor responses, and the task-repeat dynamics provides a complete description of the set of products (Thuillier et al., 2015). In addition, the PP method allows the relative importance of the sensory descriptors of the products to be established and facilitates data analysis (Valentin et al., 2012). Other reference-based methods (e.g., polarized sensory positioning or polarized projective mapping) have a critical step regarding selection of an appropriate reference sample. Once the choice of a reference product occurs within the range of products to be evaluated, PP prevents it (Ares et al., 2015; de Saldamando et al., 2015; Fleming et al., 2015).

Pivot profile shows promise, but the implementation capacity (how it is used) is still little explored and underused, and information on the procedure remains scarce. Thus, there is a need to investigate the potential application of this new method in sensory characterization of food matrices using responses directly from consumers.

The objectives of this study were (1) to investigate the use of pivot profile in the sensory characterization of commercial conventional and light or diet chocolate ice cream samples; (2) to determine the descriptive profile of these products using comment analysis of likes and dislikes provided by consumers; and (3) to compare the effectiveness of both methods in the determination of a sensory profile based on consumer perceptions.

\section{MATERIALS AND METHODS}

\section{Samples}

Six commercial samples of chocolate ice cream-3 labeled as conventional (IC1, IC2, and IC3) and 3 labeled as light or diet (ICL1, ICL2, and ICL3)were purchased from local supermarkets in the city of Campinas (São Paulo, Brazil). The specific ingredients 
are shown in Table 1. Samples were acquired and maintained under the same temperature during both storage and sensory evaluation.

\section{Sensory Evaluation}

Sensory evaluation was carried out on 2 different days at an interval of $1 \mathrm{wk}$. The tests were conducted in individual booths with adequate temperature and lighting, ensuring the comfort and privacy of panelists. The sessions were conducted in the Sensory Analysis Laboratory of the Faculty of Food Engineering, Department of Food and Nutrition, University of Campinas (Campinas, São Paulo, Brazil). Panelists were provided water and plain crackers for palate cleansing. The samples were coded with 3 random digits in monadic way and balanced for order presentation to avoid carryover effects (Macfie et al., 1989). Approval for the study was obtained from the Ethics Committee of the University of Campinas, and written consent was given by all volunteers.

Comment Analysis. One hundred twenty-three (n $=123$ ) frequent consumers of chocolate ice cream (i.e., who eat ice cream at least once a week) were recruited on campus among students, employers, and visitors, through e-mails, posters, and invitations via social networks. Participation was balanced for sex (55\% female and $45 \%$ male), with ages ranging from 18 to $50 \mathrm{yr}$. The samples were served in disposable plastic cups with a capacity of $50 \mathrm{~mL}$.

Table 1. Ingredients used in the commercial formulation (from each product's label) of different chocolate ice cream samples

\begin{tabular}{|c|c|c|c|c|c|c|}
\hline \multirow[b]{2}{*}{ Ingredient } & \multicolumn{6}{|c|}{ Ice $_{\text {cream }}{ }^{1}$} \\
\hline & $\mathrm{ICL}^{2}$ & ICL2 & $\mathrm{ICL}^{2}$ & IC1 & IC2 & $\mathrm{IC} 3^{2,3}$ \\
\hline Water & + & + & + & + & + & + \\
\hline Dry skim milk (nonfat) & + & + & + & + & - & - \\
\hline Lactitol & - & + & - & - & - & - \\
\hline Fiber & - & + & - & - & - & - \\
\hline Monoglyceride of fatty acid & + & + & + & + & + & + \\
\hline Diglycerides of fatty acid & + & - & + & + & + & + \\
\hline Carrageenan gum & + & + & + & + & + & + \\
\hline Xanthan gum & - & + & - & - & - & - \\
\hline Locust bean gum & + & + & - & + & + & - \\
\hline Sucralose & + & + & + & - & - & - \\
\hline Cocoa powder & + & + & + & - & + & + \\
\hline Dry whole milk powder & - & - & + & - & + & + \\
\hline Refined sugar & - & - & - & - & + & + \\
\hline Liquid invert sugar & - & - & - & - & + & - \\
\hline Palm oil & + & - & - & + & + & + \\
\hline Tara gum & - & - & - & - & + & - \\
\hline Guar gum & + & - & - & + & + & + \\
\hline Carboxymethyl cellulose & - & - & - & - & + & + \\
\hline Sorbitol (glucitol) & + & - & + & - & - & - \\
\hline Polydextrose & - & - & + & - & - & - \\
\hline Maltodextrin powder & - & - & + & - & - & + \\
\hline Whey & - & - & + & - & - & - \\
\hline Microcrystalline cellulose & - & - & + & - & - & - \\
\hline Carboxymethylcellulose sodium & - & - & + & - & - & - \\
\hline Crystal sugar & - & - & - & + & - & - \\
\hline Whey powder & - & - & - & + & - & - \\
\hline Glucose syrup & - & - & - & + & - & + \\
\hline Cocoa (processed with alkali) & - & - & - & + & - & - \\
\hline Liquid malt extract & - & - & - & + & - & - \\
\hline Vitamin (A, C, and D) & + & - & - & - & - & - \\
\hline Iron mineral & + & - & - & - & - & - \\
\hline Gelatin & + & - & - & - & - & - \\
\hline Demineralized whey & - & - & - & - & - & + \\
\hline Polysorbate 80 & - & - & - & - & - & + \\
\hline
\end{tabular}


The 6 chocolate ice cream samples were served to consumers in a monadic way, and consumers were asked to first assess the overall acceptance of the samples. The acceptance test used a 9-cm unstructured linear scale, anchored on the left with "dislike extremely" and on the right with "like extremely." The 9-cm unstructured scale is widely used in sensory analysis and has been successfully implemented with consumers in several studies (Esmerino et al., 2013, 2015).

After, consumers were asked to answer 2 open questions, describing in their own words the sensory attributes they liked (L) and disliked (D) in each sample. During the instruction, it was explained that answers were not mandatory, and consumers could express only likes, only dislikes, both options, and none at all for each product (Symoneaux et al., 2012). Responses were collected through the sensory software Fizz (Biosystemes, Saint-Ouen-l'Aumône, France) and then analyzed.

Pivot Profile. One hundred three $(\mathrm{n}=103)$ consumers of chocolate ice cream (60\% women), selected according to the same criteria used for comment analysis, aged 18 to $50 \mathrm{yr}$, were recruited from among students, employers, and visitors to the campus through posters, e-mails, and invitations via social networks. Around $10 \%$ of consumers had participated in the preliminary sensory studies (see Comment Analysis section), and thus learning effect was not a concern.

According to Thuillier et al. (2015), the choice of pivot results in small changes in the configurational maps generated; thus, it is not considered a critical step for good performance of the method. We chose sample IC1 as the pivot in this study.

Five pairs of samples (one pair at a time) were presented to consumers, and each pair comprised the pivot sample (sample IC1) and a coded sample, properly balanced. After receiving the pair of samples (pivot and coded sample), consumers were asked to try both samples and answer 2 open questions separately. The first question asked which sensory attributes the coded sample had in higher intensity compared with the pivot, whereas the second question asked which attributes the coded sample had in lower intensity than the pivot. It was explained to consumers that mention of sensory descriptors was not mandatory, and consumers were free to write what they considered appropriate for each sample, being asked only to avoid hedonic terms. The elicitation of terms was not mandatory because consumers can only find sensory attributes in higher intensity in the coded samples comparing to the pivot sample, and if one has to cite an attribute in lower intensity, it could be wrong. Forcing the citation of terms can lead consumers to provide words that are not appropriate to describe the samples. Data collection was carried out through the sensory software Fizz (Biosystemes), followed by textual analysis.

\section{Statistical Analysis}

Textual Analysis. For both methods, textual analysis was necessary and carefully performed. In tests using open-ended questions, each consumer is free to write without a preset format, using his or her own vocabulary. Thus, mistakes can occur and data need to be refined before being analyzed.

The terms were refined and grouped according to the following criteria: (a) verifying typing and spelling and correcting grammatical errors; (b) removing connectors and auxiliary terms when a consumer wrote a sentence instead of separate terms; (c) reducing derivatives of the same term (e.g., greasy taste), such as "greasy," "unctuous," or "oily," for example; (d) grouping a few words that were considered synonyms such as "granulosity," "presence of particles," and "lumpy," or terms referring to same intensity level, such as "slightly sweet" and "a little bit sweet." Data refinement was always based on the dictionary to identify synonyms (Symoneaux et al., 2012; Lawrence et al., 2013; Hanaei et al., 2015).

For both methods, only the modalities and descriptors mentioned by at least $5 \%$ of consumers for at least one product were used (Symoneaux et al., 2012).

After transforming the data into precise sensory terms for the comments analysis task, the descriptors were separated into modalities $\left(\mathbf{L}_{-}\right)$for likes and $\left(\mathbf{D}_{-}\right)$ for dislikes, automatically counted generating a contingency table.

The results of the pivot profile were obtained through the number of times each sensory attribute was cited as being "less intense than the pivot" (negative frequency) and "more intense than the pivot" (positive frequency). The negative frequency was subtracted from the positive frequency, resulting in a balance with different values, sometimes negative. To obtain a contingency table with positive values only, the absolute value of the lowest calculated balance of all attributes is added to all balance results. Thus, the lower value in the table was assumed zero, and all the other values were transformed into positive scores, and subsequently analyzed (Thuillier et al., 2015).

To help interpret the results, the theoretical position of the pivot (IC1) can be indicated by adding zero values for all attributes in the frequency matrix (before translation). Thus, their position and characteristics can be observed in the map generated by the correspondence analysis (CA).

Global Chi-Square and Chi-Square per Cell. As recommended by Symoneaux et al. (2012), chi-square 
analysis is an important technique for identifying statistically significant components within a matrix, besides being a suitable tool to assess the results of correlation analysis. Thus, chi-square analysis was applied to the comment analysis and pivot profile contingency tables. First, global chi-square was used to test the independence between rows and columns of each contingency table. Because significant chi-squared values were found in both analyses, it was possible to analyze and identify, within each cell, the source of the global chi-square variation through the application of chi-square per cell.

$\boldsymbol{A N O V A}$. Analysis of variance was performed on the data generated by the acceptance test, performed with the comment analysis considering the assessor and the sample as sources of variation. The averages were analyzed and significant differences were determined by Tukey's test at a significance level of $P \leq 0.05$.

Correspondence Analysis. The contingency tables containing the frequencies of each modality $(\mathrm{L})$ and (D), comment analysis, and the frequency of each translated descriptor in pivot profile were analyzed by CA (Symoneaux et al., 2012; Thuillier et al., 2015).
Similarity Between the Sensory Configurations. The regression vector coefficient (RV) using the cross-reference matrices and multiple factors analysis (MFA), based on the first 2 dimensions of the CA was used to compare and analyze similarities between the configuration derived from the 2 methods for all chocolate ice cream samples. The RV coefficient is the correlation coefficient between the 2 individual spaces of samples, ranging from 0 (totally disagree) to 1 (perfect agreement; Albert et al., 2011).

\section{RESULTS AND DISCUSSION}

\section{Comment Analysis}

The results of comment analysis and acceptance test are shown in Table 2. The attributes more appreciated and characterized as "likes" were creaminess, brown color, and chocolate aroma, followed by sweet taste, chocolate flavor, dark chocolate flavor, and melting. In contrast, the terms most frequently cited as "dislikes" were consistency, sweet taste, chocolate flavor, chocolate aroma, and brown color.

Table 2. Contingency table showing the main sensory descriptors and the frequency of use of each like and dislike comment elicited from consumers ${ }^{1}$

\begin{tabular}{|c|c|c|c|c|c|c|}
\hline \multirow[b]{2}{*}{ Attribute } & \multicolumn{6}{|c|}{ Ice cream $^{2}$} \\
\hline & ICL1 & ICL2 & ICL3 & IC1 & $\mathrm{IC} 2$ & IC3 \\
\hline \multicolumn{7}{|l|}{ Like comments } \\
\hline Brown color & 33 & 40 & 42 & 29 & 41 & 33 \\
\hline Chocolate aroma & $48^{(+)}$ & $19^{(-)}$ & 30 & 33 & $17^{(-)}$ & 31 \\
\hline Chocolate flavor & 7 & $3^{(-)}$ & 8 & 8 & 12 & $18^{(+)}$ \\
\hline Sweet taste & 11 & $2^{(-)}$ & 15 & 21 & 14 & $28^{(+)}$ \\
\hline Dark chocolate flavor & 1 & 2 & $0^{(-)}$ & 2 & 4 & 3 \\
\hline Creaminess & 45 & 39 & 42 & 37 & 44 & $53^{(+)}$ \\
\hline Melting & $0^{(-)}$ & 1 & 1 & 2 & 2 & 1 \\
\hline \multicolumn{7}{|l|}{ Dislike comments } \\
\hline Brown color & 11 & 13 & 10 & $24^{(+)}$ & 17 & 11 \\
\hline Ice crystals & 9 & 1 & 8 & 7 & 1 & 2 \\
\hline Chocolate aroma & $7^{(-)}$ & $27^{(+)}$ & 10 & 14 & $26^{(+)}$ & 11 \\
\hline Chocolate flavor & $31^{(+)}$ & 25 & 22 & 16 & $10^{(-)}$ & $5^{(-)}$ \\
\hline Sweet taste & $38^{(+)}$ & $43^{(+)}$ & 23 & 18 & 17 & $9^{(-)}$ \\
\hline Dark chocolate flavor & $2^{(-)}$ & 6 & 2 & 6 & 8 & 5 \\
\hline Dairy flavor & 3 & 2 & 4 & 2 & 1 & 1 \\
\hline Greasy taste & 3 & 1 & 3 & 4 & 3 & 2 \\
\hline Artificial sweet taste & 3 & 1 & $0^{(-)}$ & $0^{(-)}$ & 1 & $0^{(-)}$ \\
\hline Bitter & 2 & 2 & 4 & 4 & 2 & 2 \\
\hline Consistency & 30 & $47^{(+)}$ & 35 & 30 & 29 & 22 \\
\hline Melting & 1 & 1 & 3 & 1 & 4 & 1 \\
\hline Total likes (L) & 145 & $106^{(-)}$ & 138 & 132 & 134 & $167^{(+)}$ \\
\hline Total dislikes (D) & 140 & $169^{(+)}$ & 124 & 126 & 119 & $71^{(-)}$ \\
\hline Overall liking & $5.64^{\mathrm{b}}$ & $4.13^{\mathrm{c}}$ & $5.68^{\mathrm{b}}$ & $5.50^{\mathrm{b}}$ & $5.47^{\mathrm{b}}$ & $6.6^{\mathrm{a}}$ \\
\hline
\end{tabular}

${ }^{a-c}$ Means with within a row with different superscript letters differ statistically according to Tukey test $(P \leq$ $0.05)$.

${ }^{1}$ The number of citations per chocolate ice cream sample and the results of the chi-squared analysis per cell are presented. $(+)$ or $(-)$ indicate that observed values are higher or lower than the expected theoretical value. ${ }^{2} \mathrm{IC} 1, \mathrm{IC} 2$, and IC3 = conventional chocolate ice cream; ICL1, ICL2, and ICL3 = light or diet chocolate ice cream. 
We observed that the number of likes and dislikes was correlated with the acceptance test data. The higher a sample's acceptance, the greater the number of likes and the smaller the number of dislikes; less-preferred samples presented more dislikes than likes.

Table 2 shows that samples IC3 and ICL2, which had the highest and lowest scores in the acceptance test, respectively, had 167 and 106 likes versus 71 and 169 dislikes, respectively. The other samples-ICL1, IC2, ICL3, and IC1 - with intermediate scores, received similar numbers of likes and dislikes.

The chi-square test for the contingency table (Table 2) containing data from comment analysis was significant $($ chi-square value $=217.71, P<0.001)$, showing that consumers were able to accurately discriminate samples using different descriptors to justify their likes and dislikes. Thus, it was possible to apply chi-square per cell, investigating the source of variation in each cell. This improved data interpretation, allowing proper evaluation of the frequency of use of each descriptor (L or D) for one or more samples, as well as improving the observation of the sensory map.

Table 2 shows the characterization of different chocolate ice cream samples according to consumers' perceptions. The sample IC1 was characterized mainly by having a larger number of citations for brown color (D) than any other sample, probably because of the alkaline cocoa used in that formulation and different cocoa powders in the other samples. According to Saunders (2011), the type of cocoa used in the formulation affects the color and can modify viscosity and firmness of the product.

Sample IC2 received more citations for chocolate aroma (D) and fewer for chocolate aroma (L) and chocolate flavor (D) than other samples. Sample IC3 was more accepted among consumers and had the highest citation frequency for sweet taste (L), chocolate flavor (L), and creaminess (L), and fewer citations for sweet taste (D), chocolate flavor (D), and artificial sweet taste (D) between samples, the latter probably due to the absence of synthetic sweeteners in IC3.

Among the light or diet ice cream samples, sample ICL1 had higher citation frequency for chocolate aroma (L), sweet taste (D), and chocolate flavor (D) than the other samples, and fewer citations for melting (L), chocolate aroma (D), and bitter taste (D). Sample ICL3 was characterized by a lower citation frequency for dark chocolate flavor (L) and artificial sweet taste (D).

Sample ICL2 had the lowest score in the acceptance test, and it was characterized mainly by having lower citation frequency for chocolate aroma $(\mathrm{L})$ and a higher number of citations for sweet (D), chocolate flavor (D), and consistency (D).
Because perception and description of texture are shaped by physiological, socio-economic, and cultural factors (Szczesniak, 2002), some consumers were able to precisely clarify some attributes related to the texture of the ice cream samples, whereas others failed to be specific enough to distinguish the various sensory terms involved.

Consumers usually recognize fewer sensory descriptors than trained assessors, and some attributes found by consumers may overlap those used by experts in the sensory field (Brody and Lord, 2007). This may happen when using sensory methods based on consumer perception due to lack of training and vocabulary to determine specific descriptors (ten Kleij and Musters, 2003; Ares and Deliza, 2010).

Furthermore, consumers are able to express their likes and dislikes for various sensory attributes but not for all descriptors. They are good at describing simple characteristics but have some limitations to describe more complex attributes. This makes interpretation of consumers' vocabulary difficult for complex multiparameter sensations; that is, when more than one property is involved (Moskowitz et al., 1979; Moussaoui and Varela, 2010).

Whereas the term "D_consistency" had a high citation rate and separating it could lead to erroneous results, we decided to keep the term as named. As shown in Figure 1, the position of "D_consistency" is close to "D_melting," to which it could be related in this task.

After analysis of consumers' comments, the CA was applied on the data of the contingency table in order to visualize the characterization of chocolate ice cream samples in the sensory map. According to Figure 1, the first 2 dimensions of the CA accounted for $81 \%$ of the variability in the samples. The samples were similarly distributed over the first 2 dimensions and dispersed in each quadrant of the map of the CA. However, it is evident that consumers were able to distinguish the conventional and light or diet samples, which can be observed by separation of the samples from the second dimension map of CA through the descriptors D_ice crystals, D_chocolate aroma, D_sweet aftertaste, and D_bitter.

When using the data generated by the acceptance test as an additional variable, a spatial region of the overall impression is observed on the map, as well as the attributes that act positively and negatively on the acceptance of chocolate ice cream samples according to the comment analysis method.

As shown in Figure 1, chocolate flavor and creaminess were positively correlated with a product's acceptance, and consistency was negatively related to good acceptance of chocolate ice cream samples. Although 


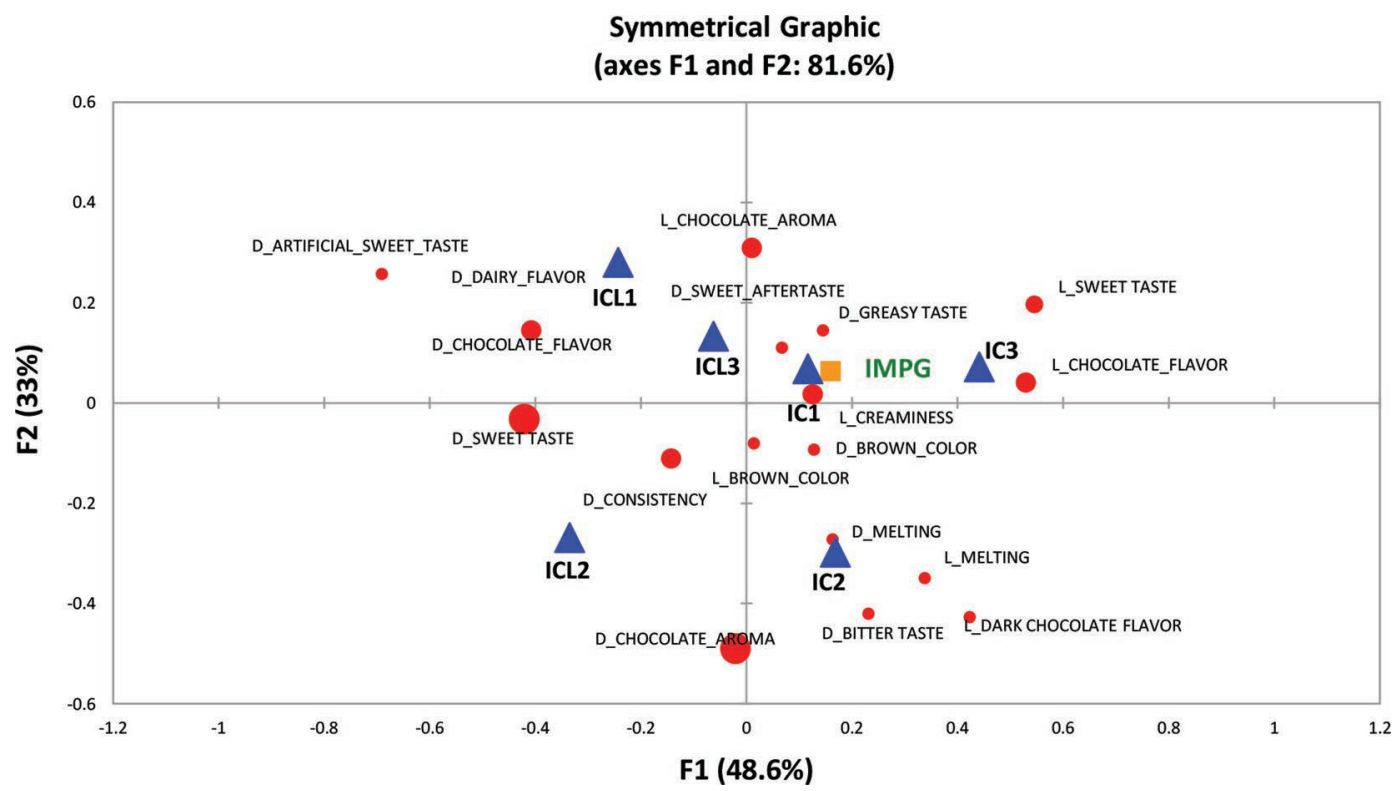

Figure 1. Correspondence map on the contingency table containing the frequency of the main modalities cited by consumers. Triangles represent the different samples and circles represent the main modalities mentioned. The different size of the circles is related to the contribution of each modality in the correspondence analysis. IC1, IC2, and IC3 = conventional chocolate ice cream; ICL1, ICL2, and ICL3 = light or diet chocolate ice cream. IMPG = overall liking. Color version available online.

sweet taste and chocolate aroma are closely related to acceptance of the samples, their origins and intensities determine the effect on product acceptance.

The use of open questions to identify drivers of preference was first suggested by ten Kleij and Musters (2003) and used by Ares and Deliza (2010) to assess key attributes for acceptance of dairy desserts. Symoneaux et al. (2012) improved the use of open questions with comment analysis of likes and dislikes and chi-square per cell as an alternative to the preference map and sensory characterization. Even though Lawrence et al. (2013) found similar results in sensory characterization conducted used free comments and classical descriptive analysis, a complex data analysis step was reported by those authors.

In the comments analysis of likes and dislikes, transcription of the sensory terms is easier when consumers are asked to answer 2 open questions compared with when only 1 open question is asked. Using comment analysis of like and dislikes, it is clearly possible to identify and differentiate the sensory attributes positively and negatively correlated with acceptance for each consumer. However, classification of the sensory attributes may be impaired during transcription of the data, once information about different intensities may be erroneously grouped and misinterpreted (Thuillier et al., 2015).
The use of chi-square per cell is noteworthy; it improved statistical analysis and in-depth observation of the comments in the contingency table, indicating the modalities and attributes more and less cited by consumers between the samples studied.

The use of chi square per cell identified the significant positioning of the modalities in the CA map, preventing misinterpretation of results through dimensional calculation artifacts (Galmarini et al., 2013). Thus, it was possible to identify that, even though the descriptor creaminess (L) is visually close to samples IC1 and ICL3, the modality was most cited and significant only for sample IC3. We also observed that samples IC2 and ICL2 significantly shared the modality chocolate aroma (D).

\section{Pivot Profile}

To begin the analysis of the data, all descriptors generated by consumers on the PP response sheets were listed. In all, 515 descriptors were obtained and carefully analyzed by researchers. After standardization of the data, the number of times that each sensory attribute was more and less cited than the pivot (positive and negative frequency, respectively) was automatically counted and listed (Table 3 ). The visualization 
Table 3. Frequency example observed for each attribute in ICL1 and ICL2 samples in pivot profile method ${ }^{1}$

\begin{tabular}{llcrrr}
\hline Sample $^{2}$ & Attribute & $\begin{array}{c}\text { Frequency } \\
\text { positive }\end{array}$ & $\begin{array}{c}\text { Frequency } \\
\text { negative }\end{array}$ & Balance & $\begin{array}{c}\text { Translated } \\
\text { frequency }\end{array}$ \\
\hline ICL1 & Sweet taste & 48 & -26 & 22 & 93 \\
ICL1 & Creaminess & 17 & -16 & 1 & 72 \\
ICL1 & Dairy flavor & 16 & -7 & 9 & 80 \\
ICL1 & Consistency & 1 & -3 & -2 & 69 \\
ICL1 & Milk aroma & 0 & -2 & -2 & 69 \\
ICL1 & Chocolate aroma & 27 & -11 & 16 & 87 \\
ICL1 & Firmness & 3 & -6 & -3 & 68 \\
ICL1 & Ice crystals & 4 & -5 & -1 & 70 \\
ICL1 & Brown color & 40 & -2 & 38 & 109 \\
ICL1 & Chocolate flavor & 20 & -38 & -18 & 53 \\
ICL1 & Artificial chocolate flavor & 4 & -1 & 3 & 74 \\
ICL1 & Sweet aroma & 3 & -2 & 1 & 72 \\
ICL2 & Sweet taste & 1 & -72 & -71 & 0 \\
ICL2 & Creaminess & 19 & -4 & 15 & 86 \\
ICL2 & Dairy flavor & 11 & -9 & 2 & 73 \\
$\ldots$ & Consistency & 3 & -1 & 2 & 73 \\
\hline
\end{tabular}

${ }^{1}$ The number of citations for "less" (negative frequency), "more" (positive frequency), balance (the difference between positive and negative), and the translated frequency are presented. (...) indicates that there are more samples and their frequency observed in the table.

${ }^{2}$ ICL1 and ICL2 = light or diet chocolate ice cream samples.

of positive and negative frequencies and the translated frequency gives an idea of the intensity of the attributes mentioned compared with those of the pivot.

According to Table 3, among samples labeled as light or diet, sample ICL1 was perceived mainly as browner, having more intense chocolate aroma and sweet taste, and with less chocolate flavor than the pivot. In contrast, sample ICL2 was mainly characterized by having less sweet taste and chocolate flavor, and more bitter taste, brown color, and creaminess than the pivot.

Sample ICL3 had less intense sweet taste and chocolate flavor, and more intense brown color, chocolate aroma, and milky flavor than the pivot (data not shown). It is noteworthy that IC3 was characterized mainly by having higher milky flavor, chocolate flavor, creaminess, and lower ice crystals than the pivot. According to Severini et al. (2008), the presence of sugars in the formulation gives greater stability to the product, once it reduces water activity, thus preventing the formation of ice crystals. Compared with sample IC2, the greatest difference was the greater intensity of brown color and dark chocolate flavor, and less intense sweet taste.

After transformation of data into positive values (i.e., frequency translated), a contingency table for the pivot profile was formed (Table 4). Correspondence analysis was applied to the table, creating a sensory map of the spatial configuration of the samples according to their characteristics, as shown in Figure 2. In addition, the chi-square test was applied to Table 4 , and statistical significance was observed $\left(\chi^{2}=196.46, P<0.001\right)$ among the values, showing that consumers were able to adequately differentiate the samples. From this, by us- ing the chi-square per cell, we were able to investigate the source of variation within each cell that makes up a data matrix, and identify the descriptors that were statistically more and less cited than the pivot.

According to Figure 2, the first 2 dimensions of CA accounted for $91.3 \%$ of the variability among the chocolate ice cream samples. Through the CA map and with the help of chi-square per cell, it was clear that the samples IC2 and ICL2 received more citations for brown color compared with the other samples, whereas sample IC3 received fewer citations. The attribute chocolate flavor was more pronounced for samples IC2 and IC3, as well as sweet taste for samples ICL1 and IC3. Importantly, IC2 received more individual citations for dark chocolate and milky flavors.

In the pivot profile, the term "consistency" was more related to samples ICL2, ICL3, and IC2. It was subjectively cited by consumers and the researchers decided to maintain it for the same reason described above for comment analysis. The term was close to other terms such as "firmness" and "melting," indicating a possible correlation with these descriptors.

Our findings corroborate some previous studies on ice cream with fat and sugar replacers, in which hardness and firmness significantly increased (Cadena et al., 2012; Javidi et al., 2016). It is expected that ice creams containing fat will melt more slowly than the nonfat ice creams, as observed in the present study, because the rate of heat transfer through the ice cream base is lower (Li et al., 1997; Warren and Hartel, 2014).

Although sample IC2 was a conventional formulation, the results obtained may be due to the use of some 


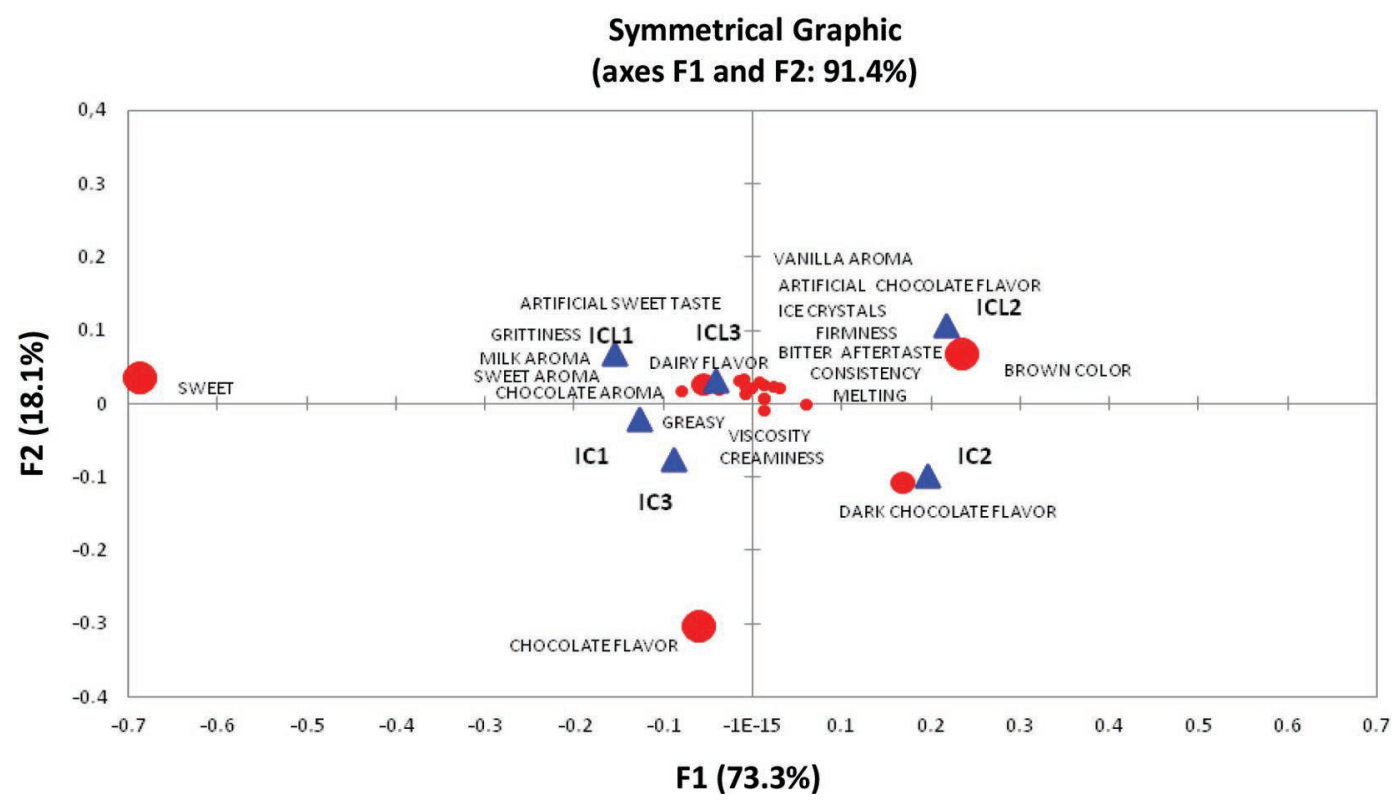

Figure 2. Correspondence map on the contingency table containing the translated frequency of the main sensory attributes cited by consumers. Triangles represent the different samples and circles represent the main modalities mentioned. The different size of the circles is related to the contribution of each modality in the correspondence analysis. IC1, IC2, and IC3 = conventional chocolate ice cream; ICL1, ICL2, and ICL3 $=$ light or diet chocolate ice cream. Color version available online.

hydrocolloids. According to Soukoulis et al. (2008), hardness of regular ice creams increases with addition of xanthan, guar, carboxymethylcellulose, and sodium alginate, because of the control of recrystallization through enhancement of microviscosity and cryogelation.

The texture attribute "creaminess" is a complex sensory attribute directly related to the overall acceptabil-

Table 4. Contingency table containing the translated frequency of the main attributes indicated by consumers in pivot profile task

\begin{tabular}{|c|c|c|c|c|c|}
\hline \multirow[b]{2}{*}{ Attribute } & \multicolumn{5}{|c|}{ Ice $_{\text {cream }}^{2}$} \\
\hline & ICL1 & ICL2 & ICL3 & $\mathrm{IC} 2$ & IC3 \\
\hline Brown color & 109 & $140^{(+)}$ & 101 & $147^{(+)}$ & $77^{(-)}$ \\
\hline Ice crystals & 70 & 66 & 68 & 66 & 61 \\
\hline Viscosity & 67 & 70 & 72 & 73 & 73 \\
\hline Chocolate aroma & 87 & 59 & 83 & 71 & 71 \\
\hline Sweet aroma & 72 & 66 & 70 & 64 & 71 \\
\hline Vanilla aroma & 71 & 71 & 71 & 69 & 70 \\
\hline Milk aroma & 69 & 68 & 71 & 68 & 69 \\
\hline Chocolate flavor & 53 & $29^{(-)}$ & 59 & $89^{(+)}$ & $88^{(+)}$ \\
\hline Sweet taste & $93^{(+)}$ & $0^{(-)}$ & 56 & $7^{(-)}$ & $63^{(+)}$ \\
\hline Dark chocolate flavor & $69^{(-)}$ & 84 & 74 & $122^{(+)}$ & 80 \\
\hline Dairy flavor & 80 & 73 & 88 & $66^{(+)}$ & 88 \\
\hline Artificial chocolate flavor & 74 & 74 & 71 & 71 & 71 \\
\hline Greasy taste & 72 & 74 & 74 & 74 & 75 \\
\hline Bitter aftertaste & 71 & 74 & 74 & 71 & 71 \\
\hline Sweet aftertaste & 76 & 71 & 72 & 71 & 71 \\
\hline Grittiness & 74 & 70 & 69 & 67 & 69 \\
\hline Creaminess & 72 & 86 & 80 & 84 & 85 \\
\hline Melting & 71 & 72 & 70 & 73 & 72 \\
\hline Firmness & 68 & 77 & 77 & 73 & 73 \\
\hline Consistency & 69 & 73 & 77 & 73 & 67 \\
\hline
\end{tabular}

${ }^{1}$ The translated frequency and the results of chi-square analysis per cell are presented. $(+)$ or $(-)$ indicate that observed values are higher or lower than the expected theoretical value.

${ }^{2} \mathrm{IC} 2$, and IC3 = conventional chocolate ice cream; ICL1, ICL2, and ICL3 = light or diet chocolate ice cream. Because IC1 was used as the pivot sample, it is not shown in the table. 
Coordinates of the partial clouds (axes F1 and F2: 97.9\%)

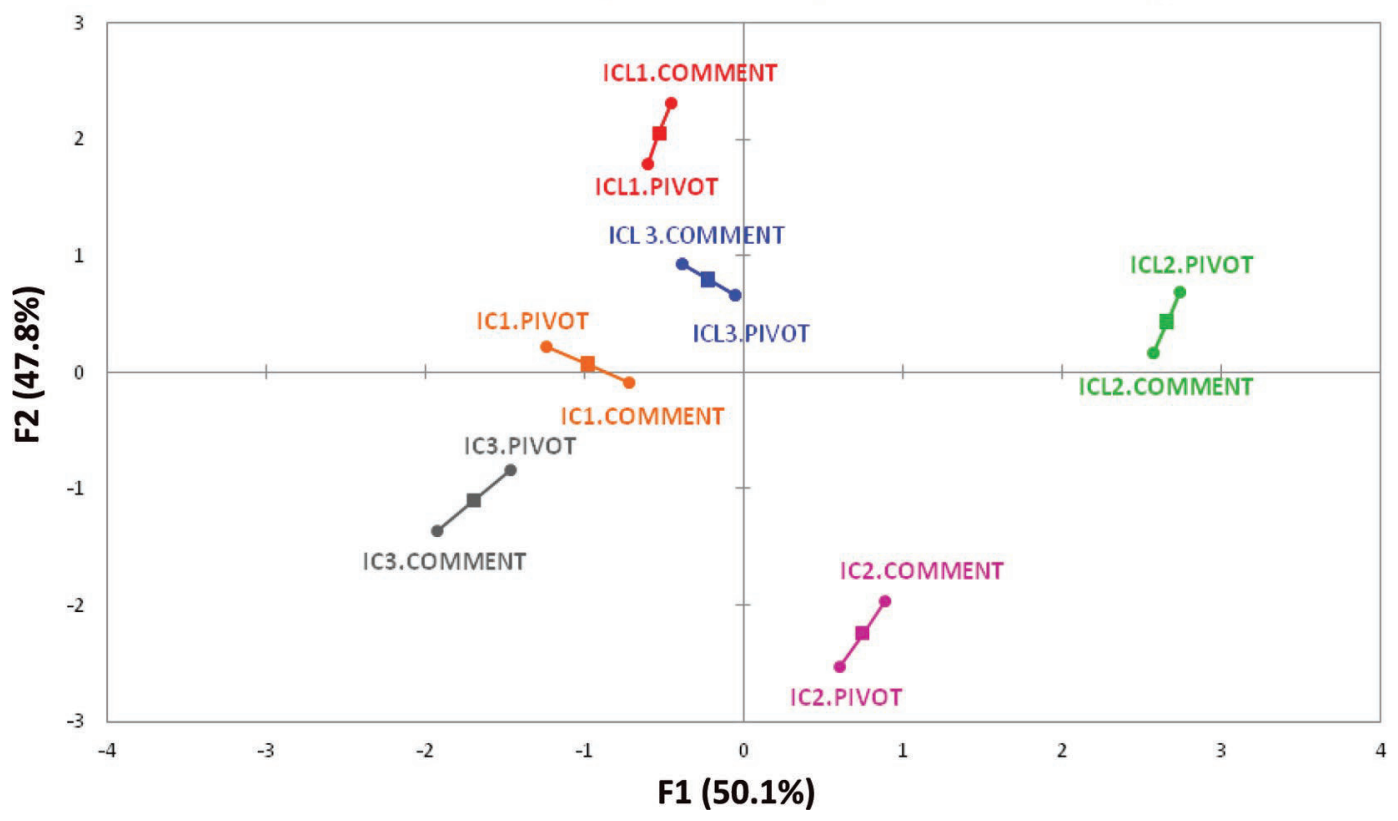

Figure 3. Comparative configurational map generated by multiple factor analysis over individual configuration of comment analysis and pivot profile methods for chocolate ice cream samples. IC1, IC2, and IC3 = conventional chocolate ice cream; ICL1, ICL2, and ICL3 = light or diet chocolate ice cream. Color version available online.

ity of semi-solid dairy desserts like ice cream. "Creamy" is a term used by consumers to describe several characteristics, often without clear distinctions between them (Kirkmeyer and Tepper, 2003). As can be seen in the present findings and in accordance with previous studies, this attribute is particularly influenced by compositional parameters such as hydrocolloids, fat, and sugars (Soukoulis and Tzia, 2010; Warren and Hartel, 2014).

It is worth noting the distinction between samples made using the PP method, in which the conventional and light or diet samples were separated by the first factor, indicating the significant difference between samples and the sensitive perception of consumers.

\section{Similarity Between the Sensory Configurations}

The last step of the study was to compare the configurational similarity of the samples as perceived by consumers using 2 different methods. Visualization was carried out through the application of MFA on the cross-tab containing the frequency of each modality (comment analysis) and the translated frequency (pivot profile; Cruz et al., 2013; Reinbach et al., 2014).

Figure 3 shows the first 2 dimensions of the MFA map, with almost $98 \%$ of variance explained. Each sample was represented by a different color, and the evaluation was indicated in the generated map according to the method. A clear distinction among samples can be observed, demonstrating that consumers were able to differentiate one sample from another.

When comparing the sensory characteristics generated by both methodologies using the RV coefficient, a great similarity between methods was observed ( $\mathrm{RV}=$ 0.917). Reinbach et al. (2014), in a comparative study, found high similarity between 2 variants of the check-allthat-apply (CATA) method $(\mathrm{RV}=0.97)$, and between Napping and CATA $(R V=0.93)$. Symoneaux et al. (2012) compared the results of comment analysis with those obtained by the assessors trained in QDA, and found consistency between the results, demonstrating the validity and reliability of the methodology. However, little is known about the validity and robustness of the pivot profile method.

According to our results, both methods were equally effective in sensory characterization of commercial chocolate ice cream. Once comment analysis was able to display the drivers of liking, with high similarity with the results, it was possible to hypothesize similar results in the pivot profile. However, further studies considering hedonic ratings associated with pivot profile should be carried out. Despite having different cognitive demands, the results produced by both methods were very similar, due to the intrinsic differences of each commercial formulation and associations between the higher or lower intensity of some attributes and the products' acceptance. 
According to Booth and Conner (1990), in some foods, the lowest intensity of certain characteristics such as offflavor has a positive effect on the product's acceptance, as well as the high intensity of positive characteristics. In these monotonic cases, a linear regression can be directly used to identify the psychophysical function of acceptance. However, for many other sensory attributes, each consumer has a particular preference level for a particular food. Products with high intensity of these attributes or with lower intensity compared with an ideal product will lose product acceptance.

Kahneman (2011) proposed 2 different modes of thinking that are involved in consumers' perceptions, known as system 1 and system 2 . System 1 is the model used in comment analysis, and consists of an affective, associative, and experience-based approach, characterized by dynamic and automatic responses, without requiring mental concentration (Lieberman, 2000; Morewedge and Kahneman, 2010). In contrast, system 2 is rational, analytical, and deliberative, being responsible for processing information slowly and in detail (Stanovich and West, 2000, Toplak et al., 2010; Ares et al., 2014). The pivot profile method seems to be more consistent with the predominance of thoughtcontrolled system 2.

According to Pennycook et al. (2015), the mechanism for the prevalence of one system of thought over the other is not very clear. Some authors believe that both systems work together, system 2 being linked to system 1 (Stanovich and West, 2000; Ares et al., 2014). System 1, responsible for fast and autonomous processes, is prevalent when one needs rapid standard responses. If that process is overridden by the need for more analytical reasoning, system 2 may prevail, requiring hypothetical thinking and memory usage in the deepest way (Evans and Stanovich, 2013).

When the discriminative power of the methods was evaluated, a cognitive differentiation can be easily observed. Whereas comment analysis was able to identify only 13 major sensory attributes among its modalities (L) and (D), pivot profile demonstrated higher performance in the sensory characterization of the samples, being able to identify 20 different main descriptors.

Studies on acceptance tests have shown that consumers integrate information from stimuli received by food and assign a global value for each sample ( $\mathrm{Su}$ et al., 2012). Acceptance may be defined as a process of close engagement with the present moment (Dan-Glauser and Gross, 2015), highly related to emotional situations (Wolgast et al., 2011) and automatic responses (Alberts et al., 2012). It is strongly influenced by sensory expectations (related to ideas about sensory characteristics) and hedonic expectations (related to the desire for the product) (Varela et al., 2010). According to Ares (2015), understanding the cognitive processes involved in the performance of emerging methodologies of sensory characterization using consumers could bring great contribution for their implementation and dissemination. When acceptance analysis alone is insufficient for the development of new products and reformulation of existing products (Spinelli et al., 2014), more objective methods such as pivot profile may be used in sensory characterization studies.

\section{CONCLUSIONS}

Both the comment analysis and pivot profile methods were able to characterize different chocolate ice cream samples using consumers' perceptions. The chi-square analysis per cell allowed us to highlight significant differences between samples according to the method used. Although comment analysis has shown good results and correlations with pivot profile, the comment analysis method is performed intuitively by consumers. Its results can be variable and should be evaluated with caution before making decision. Pivot profile is an important and promising tool for sensory analysis. Compared with other open-ended questions for sensory evaluation, it has high analytical and discriminative power, making it useful in routine applications when classical descriptive methods cannot be applied. Future studies are needed to develop best methodological practices. Questions remain regarding the minimum number of assessors, use of different pivot samples in the same study, inclusion of hedonic evaluations, and comparisons with classic descriptive analyses.

\section{ACKNOWLEDGMENTS}

We thank Kalinca Cusielo (University of Campinas, Faculty of Food Engineering, Department of Food and Nutrition, Campinas, São Paulo, Brazil) for her assistance and collaboration in the present work.

\section{REFERENCES}

Albert, A., P. Varela, A. Salvador, G. Hough, and S. Fiszman. 2011. Overcoming the issues in the sensory description of hot served food with a complex texture. Application of QDA ${ }^{\circledR}$, flash profiling and projective mapping using panels with different degrees of training. Food Qual. Prefer. 22:463-473.

Alberts, H. J. E. M., F. Schneider, and C. Martijn. 2012. Dealing efficiently with emotions: Acceptance-based coping with negative emotions requires fewer resources than suppression. Cogn. Emot. $26: 863-870$.

Ares, G. 2015. Methodological challenges in sensory characterization. Curr. Opin. Food Sci. 3:1-5.

Ares, G., L. Antúnez, D. Oliveira, F. Alcaire, A. Giménez, I. Berget, T. Næs, and P. Varela. 2015. Pole selection in polarized sensory 
positioning: Insights from the cognitive aspects behind the task. Food Qual. Prefer. 46:48-57.

Ares, G., and R. Deliza. 2010. Studying the influence of package shape and colour on consumer expectations of milk desserts using word association and conjoint analysis. Food Qual. Prefer. 21:930-937.

Ares, G., F. Mawad, A. Giménez, and A. Maiche. 2014. Influence of rational and intuitive thinking styles on food choice: Preliminary evidence from an eye-tracking study with yogurt labels. Food Qual. Prefer. 31:28-37.

Aykan, V., E. Sezgin, and Z. B. Guzel-Seydim. 2008. Use of fat replacers in the production of reduced-calorie vanilla ice cream. Eur. J. Lipid Sci. Technol. 110:516-520.

Bécue-Bertaut, M. 2014. Tracking verbal-based methods beyond conventional descriptive analysis in food science bibliography. A statistical approach. Food Qual. Prefer. 32(A):2-15.

Booth, D. A., and M. T. Conner. 1990. Characterisation and measurement of influences on food acceptability by analysis of choice differences: Theory and practice. Food Qual. Prefer. 2:75-85.

Brody, A. L., and J. B. Lord. 2007. Developing New Food Products for a Changing Marketplace. 2nd ed. CRC Press, Boca Raton, FL.

Cadena, R. S., and H. M. A. Bolini. 2011. Time-intensity analysis and acceptance test for traditional and light vanilla ice cream. Food Res. Int. 44:677-683.

Cadena, R. S., A. G. Cruz, J. A. F. Faria, and H. M. A. Bolini. 2012. Reduced fat and sugar vanilla ice creams: Sensory profiling and external preference mapping. J. Dairy Sci. 95:4842-4850.

Cruz, A. G., A. E. C. Antunes, A. L. O. P. Sousa, J. A. F. Faria, and S. M. I. Saad. 2009. Ice-cream as a probiotic food carrier. Food Res. Int. 42:1233-1239.

Cruz, A. G., R. S. Cadena, W. F. Castro, E. A. Esmerino, J. B. Rodrigues, L. Gaze, J. A. F. Faria, M. Q. Freitas, R. Deliza, and H. M. A. Bolini. 2013. Consumer perception of probiotic yogurt: Performance of check all that apply (CATA), projective mapping, sorting and intensity scale. Food Res. Int. 54:601-610.

da Silva, V. M., V. P. R. Minim, M. A. M. Ferreira, P. H. P. Souza, L. E. S. Moraes, and L. A. Minim. 2014. Study of the perception of consumers in relation to different ice cream concepts. Food Qual. Prefer. 36:161-168.

Dan-Glauser, E. S., and J. J. Gross. 2015. The temporal dynamics of emotional acceptance: Experience, expression, and physiology. Biol. Psychol. 108:1-12.

de Saldamando, L., L. Antúnez, M. Torres-moreno, A. Giménez, and G. Ares. 2015. Reliability of polarized projective mapping with consumers. J. Sens. Stud. 30:280-294.

Dervisoglu, M., and F. Yazici. 2006. Note. The effect of citrus fibre on the physical, chemical and sensory properties of ice cream. Food Sci. Technol. Int. 12:159-164.

Esmerino, E. A., A. G. Cruz, E. P. R. Pereira, J. B. Rodrigues, J. A. F. Faria, and H. M. A. Bolini. 2013. The influence of sweeteners in probiotic petit suisse cheese in concentrations equivalent to that of sucrose. J. Dairy Sci. 96:5512-5521.

Esmerino, E. A., J. A. Paixão, A. G. Cruz, L. Garitta, G. Hough, and H. M. A. Bolini. 2015. Survival analysis: A consumer-friendly method to estimate the optimum sucrose level in probiotic petit suisse. J. Dairy Sci. 98:7544-7551.

Evans, J. S., and K. E. Stanovich. 2013. Dual-process theories of higher cognition: Advancing the debate. Perspect. Psychol. Sci. $8: 223-241$.

Ferraz, J. L., A. G. Cruz, R. S. Cadena, M. Q. Freitas, U. M. Pinto, C. C. Carvalho, J. A. F. Faria, and H. M. A. Bolini. 2012. Sensory acceptance and survival of probiotic bacteria in ice cream produced with different overrun levels. J. Food Sci. 77:S24-S28.

Fleming, E. E., G. R. Ziegler, and J. E. Hayes. 2015. Check-all-thatapply (CATA), sorting, and polarized sensory positioning (PSP) with astringent stimuli. Food Qual. Prefer. 45:41-49.

Frøst, M. B., H. Heymann, W. L. P. Bredie, G. B. Dijksterhuis, and M. Martens. 2005. Sensory measurement of dynamic flavour intensity in ice cream with different fat levels and flavourings. Food Qual. Prefer. 16:305-314.

Galmarini, M. V., R. Symoneaux, S. Chollet, and M. C. Zamora. 2013. Understanding apple consumers' expectations in terms of likes and dislikes. Use of comment analysis in a cross-cultural study. Appetite $62: 27-36$.

Giboreau, A., C. Dacremont, S. Guerrand, and D. Dubois. 2009. Décrire: Identifier ou catégoriser? Page 211-232 in Le Sentir et le Dire. D. Dubois, ed. L'Harmattan, Paris, France.

Hanaei, F., G. Cuvelier, and J. M. Sieffermann. 2015. Consumer texture descriptions of a set of processed cheese. Food Qual. Prefer. 40(B):316-325.

Javidi, F., S. M. A. Razavi, F. Behrouzian, and A. Alghooneh. 2016. The influence of basil seed gum, guar gum and their blend on the rheological, physical and sensory properties of low fat ice cream. Food Hydrocoll. 52:625-633.

Kahneman, D. 2011. Thinking, Fast and Slow. Farrar, Straus and Giroux, New York, NY.

King, S. C., H. L. Meiselman, and B. T. Carr. 2010. Measuring emotions associated with foods in consumer testing. Food Qual. Prefer. 21:1114-1116.

Kirkmeyer, S. V., and B. J. Tepper. 2003. Understanding creaminess perception of dairy products using free-choice profiling and genetic responsivity to 6-n-propylthiouracil. Chem. Senses 28:527-536.

Lawrence, G., R. Symoneaux, I. Maitre, F. Brossaud, M. Maestrojuan, and E. Mehinagic. 2013. Using the free comments method for sensory characterisation of Cabernet Franc wines: Comparison with classical profiling in a professional context. Food Qual. Prefer. 30:145-155.

Li, Z., R. Marshall, H. Heymann, and L. Fernando. 1997. Effect of milk fat content on flavor perception of vanilla ice cream. J. Dairy Sci. 80:3133-3141.

Lieberman, M. D. 2000. Intuition: A social cognitive neuroscience approach. Psychol. Bull. 126:109-137.

Macfie, H. J., N. Bratchell, K. Greenhoff, and L. V. Vallis. 1989. Designs to balance the effect of order of presentation and first-order carry-over effects in hall tests. J. Sens. Stud. 4:129-148.

Meilgaard, M., G. V. Civille, and B. T. Carr. 1999. Sensory Evaluation and Techniques. 3rd ed. CRC Press LLC, Boca Raton, FL.

Morewedge, C. K., and D. Kahneman. 2010. Associative processes in intuitive judgment. Trends Cogn. Sci. 14:435-440.

Moskowitz, H. R., J. C. Kapsalis, A. V. Cardello, D. Fishken, O. Maller, and R. A. Segars. 1979. Determining relationships among objective, expert, and consumer measures of texture. Food Technol. $33: 84-88$

Moussaoui, K. A., and P. Varela. 2010. Exploring consumer product profiling techniques and their linkage to a quantitative descriptive analysis. Food Qual. Prefer. 21:1088-1099.

Ozdemir, C., A. Arslaner, S. Ozdemir, and M. Allahyari. 2015. The production of ice cream using stevia as a sweetener. J. Food Sci. Technol. 52:7545-7548.

Parente, M. E., A. V. Manzoni, and G. Ares. 2011. External preference mapping of commercial antiaging creams based on consumers' responses to a check-all-that-apply question. J. Sens. Stud. $26: 158-166$.

Pennycook, G., J. A. Fugelsang, and D. J. Koehler. 2015. What makes us think? A three-stage dual-process model of analytic engagement. Cognit. Psychol. 80:34-72.

Reinbach, H. C., D. Giacalone, L. M. Ribeiro, W. L. P. Bredie, and M. B. Frøst. 2014. Comparison of three sensory profiling methods based on consumer perception: CATA, CATA with intensity and Napping ${ }^{\circledR}$. Food Qual. Prefer. 32(B):160-166.

Saunders, A. B. 2011. Ice Cream and Desserts | Dairy Desserts. Pages 905-912 in Encyclopedia of Dairy Sciences. 2nd ed. J. W. Fuquay, ed. Academic Press, San Diego, CA.

Severini, C., M. R. Corbo, A. Derossi, A. Bevilacqua, and R. Giuliani. 2008. Use of humectants for the stabilization of pesto sauce. Int. J. Food Sci. Technol. 43:1041-1046.

Soukoulis, C., I. Chandrinos, and C. Tzia. 2008. Study of the functionality of selected hydrocolloids and their blends with $\kappa$-carrageenan on storage quality of vanilla ice cream. Food Sci. Technol. (Campinas) 41:1816-1827.

Soukoulis, C., and C. Tzia. 2010. Response surface mapping of the sensory characteristics and acceptability of chocolate ice cream containing alternate sweetening agents. J. Sens. Stud. 25:50-75. 
Spinelli, S., C. Masi, C. Dinnella, G. P. Zoboli, and E. Monteleone 2014. How does it make you feel? A new approach to measuring emotions in food product experience. Food Qual. Prefer. 37:109122.

Stanovich, K. E., and R. F. West. 2000. Individual differences in reasoning: implications for the rationality debate? Behav. Brain Sci. 23:645-665.

Su, Y., L.-L. Rao, X. Li, Y. Wang, and S. Li. 2012. From quality to quantity: The role of common features in consumer preference. J. Econ. Psychol. 33:1043-1058.

Sun-Waterhouse, D., L. Edmonds, S. S. Wadhwa, and R. Wibisono. 2013. Producing ice cream using a substantial amount of juice from kiwifruit with green, gold or red flesh. Food Res. Int. 50:647-656.

Symoneaux, R., M. V. Galmarini, and E. Mehinagic. 2012. Comment analysis of consumer's likes and dislikes as an alternative tool to preference mapping. A case study on apples. Food Qual. Prefer. $24: 59-66$

Szczesniak, A. S. 2002. Texture is a sensory property. Food Qual. Prefer. 13:215-225.

ten Kleij, F., and P. A. D. Musters. 2003. Text analysis of open-ended survey responses: A complementary method to preference mapping. Food Qual. Prefer. 14:43-52.
Thuillier, B., D. Valentin, R. Marchal, and C. Dacremont. 2015. Pivot $\left.{ }^{(}\right)$profile: A new descriptive method based on free description. Food Qual. Prefer. 42:66-77.

Toplak, M. E., G. B. Sorge, A. Benoit, R. F. West, and K. E. Stanovich. 2010. Decision-making and cognitive abilities: A review of associations between Iowa Gambling Task performance, executive functions, and intelligence. Clin. Psychol. Rev. 30:562-581.

Valentin, D., S. Chollet, M. Lelièvre, and H. Abdi. 2012. Quick and dirty but still pretty good: A review of new descriptive methods in food science. Int. J. Food Sci. Technol. 47:1563-1578.

Varela, P., G. Ares, A. Giménez, and A. Gámbaro. 2010. Influence of brand information on consumers' expectations and liking of powdered drinks in central location tests. Food Qual. Prefer. 21:873880.

Warren, M. M., and R. W. Hartel. 2014. Structural, compositional, and sensorial properties of United States commercial ice cream products. J. Food Sci. 79:E2005-E2013. 